\title{
Dielectric Loss Factor Measurement of High Voltage Capacitive Apparatus Based on Harmonic Analysis Method
}

\author{
Qiang Gao ${ }^{1,2}$, Baohong Geng ${ }^{2}$, Guangming Zhang ${ }^{2}$, Yadong Liu ${ }^{1}$, Feng Yuan ${ }^{2}$, \\ Gehao Sheng ${ }^{1}$ and Xiuchen Jiang ${ }^{1}$ \\ ${ }^{1}$ Shanghai Jiao Tong University, China \\ ${ }^{2}$ Electric Power Research Institute, State Grid Liaoning Electric Power Supply Co. \\ Ltd., Shenyang China \\ * Corresponding author: garygq@gmail.com
}

\begin{abstract}
Dielectric loss factor measurement is an important index in accessing the insulation performance of power equipment. With the development of the condition-based maintenance and smart grid, more attention has been paid to online diagnostic technologies for the dielectric loss factor measurement. Various methods have been developed in this field in which harmonic analysis (HA) is prevalent due to its performance of resisting disturbance and stability superior to others. When performing the HA method on the sampled data, the non-simultaneous sampling and the lack of synchronization between sampling rate and the signal period are believed to be the main source of error in the measurement of the magnitude and the phase of the signals. To improve the effectiveness of the HA method, measures were taken by means of the designing online monitoring system. The basic principle of the HA method is presented in this paper and an online monitoring system based on DSP technology is developed accordingly. At last, this paper analyses the on-line monitoring results resulted from the online monitoring system on a substation.
\end{abstract}

Keywords: Dielectric Loss Factor, Harmonic Analysis Method, Online Monitoring System

\section{Introduction}

To ensure the safe and reliable operation of a power system it is necessary to monitor periodically the insulation condition of $\mathrm{HV}$ apparatus. This may be accomplished through measuring partial discharge levels, dielectric loss factor and capacitance using conventional well-established time based maintenance method. To implement these routine tests require removal of equipment from service, however, the measure and continuous monitor may be carried out directly if on-line monitoring method is adopted. These on-line dielectric loss factor measuring methods detect the current flowing through the test object and the voltage signal applied to it. The various techniques reported in the literature differ from each other in the manner how the signals are acquired and how the gathered data are handled. In the work reported in this paper a distributed on-line monitoring system has been developed based on digital signal processing (DSP) technology. A method based on harmonic analysis using Discrete Fourier transform (DFT) has been used. To 
improve the effectiveness of the DFT algorithm, a few difficulties were overcome when the most fundamental method is utilized in digital spectrum analysis.

The on-line monitoring system detect the following parameters: running voltage, frequency, insulation current (current pass through capacitance of equipment), capacitance, dielectric loss factor $\tan \delta$, the current and voltage harmonic components of the 3rd, 5th and 7th, environment temperature and humidity etc.

\section{Principle of Measurement Based on Harmonic Analysis Method}

For the high-voltage capacitive apparatus and the metal oxide arrester (MOA), the equivalent circuit of parallel connection of $\mathrm{R}$ and $\mathrm{C}$ (shown in Figure 1 and Figure 2) can be used to analyze the measuring principle. It is assumed that $u(t)$ is the running voltage of the apparatus, $i(t)$ is the leakage current, $i_{r}$ and $i_{c}$ is the resistance and capacitance current respectively.

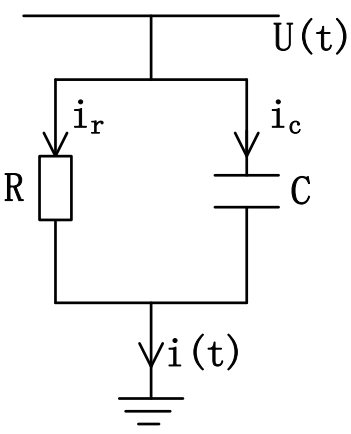

Figure 1. Equivalent Circuit

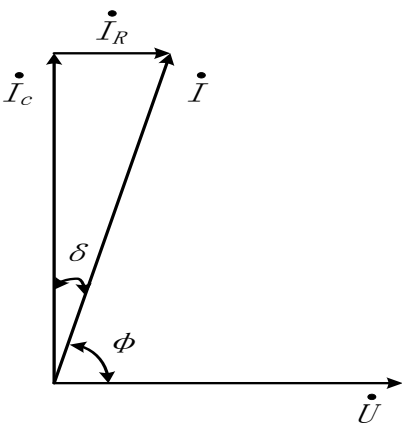

Figure 2. Vector Graph

Suppose $u(t)$ and $i(t)$ are simultaneously and continuously measured voltage and current signals from one phase of the power equipment, then their Fourier series can be written in a general form as follow.

$$
f(t)=a_{0}+\sum_{n=1}^{\infty}\left(a_{n} \cos \left(n \omega_{0} t\right)+b_{n} \sin \left(n \omega_{0} t\right)\right)
$$

Where $a_{0}$ is the dc component of the $f(t)$ signal. The harmonics of this $f(t)$ signal have frequencies $n \omega_{0}$, where $\omega_{0}$ is the fundamental frequency and $\mathrm{n}=1,2$, $3, \ldots$ Through Fourier analysis, the constants $\omega_{0}, a_{0}, a_{n}, b_{n}$ can be determined by the following equations.

$$
\begin{gathered}
\omega_{0}=2 \pi / T \\
a_{0}=\frac{1}{T} \int_{0}^{T} f(t) d t \\
a_{n}=\frac{2}{T} \int_{0}^{T} f(t) \cos n \omega_{0} t d t
\end{gathered}
$$




$$
\begin{gathered}
b_{n}=\frac{2}{T} \int_{0}^{T} f(t) \sin n \omega_{0} t d t \\
A_{n}=\sqrt{a_{n}^{2}+b_{n}^{2}} \\
\varphi_{n}=\arctan \left(-b_{n} / a_{n}\right) \\
\delta=\pi / 2-\left(\varphi_{i}-\varphi_{u}\right) \\
I_{r}=I \times \cos (\pi / 2-\delta) \\
I_{c}=I \times \sin (\pi / 2-\delta)
\end{gathered}
$$

The amplitude of the nth harmonics at a frequency $n \omega_{0}$ can be calculated through equation (6). In addition to the amplitude, the phase angle $\varphi_{n}$ can be calculated by equation (7). A current transducer and a voltage transducer capture the leakage current and running voltage of the apparatus respectively. The two analog signals then converted into digital form by an A/D converter, through the DFT transformation the fundamental component of the waveform can be extracted, thus the dielectric loss factor and capacitance of the high voltage apparatus are capable to be obtained. The fundamental amplitude of the voltage and current

Signals $U, I$ and the fundamental phase angles $\varphi_{u}, \varphi_{i}$ can be calculated through the above equations. Finally, the dielectric loss angle can be directly expressed by equation (8). As for the metal oxide arrester, the resistance current and capacitance current can be calculated through the equation (9) and (10) respectively.

\section{Design of the Online Monitoring System}

The distributed on-line monitoring system reported in this paper is based on the half duplex RS-485 bus and fiber optical communication technology as shown in Figure 3. The monitoring system consists of three parts: a) local control unit, b) substation control unit, c) remote expert and diagnosis platform. This paper only focuses on the introduction of the local control unit and substation control unit. As the remote expert and diagnosis platform is still under construction, this paper will not present more information about it.

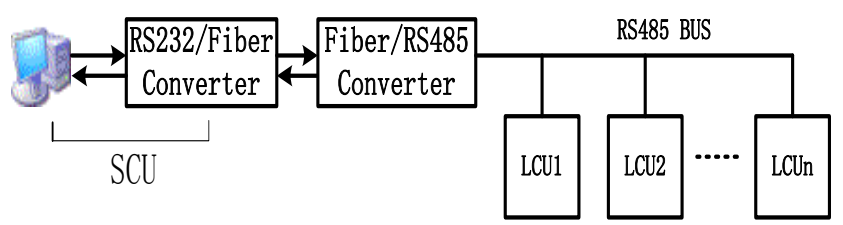

Figure 3. Schematic Diagram of the Distributed Online Monitoring System 


\subsection{Local Control Unit}

Local control unit (LCU) is the key component of the substation online monitoring system. To reduce the signal and power supply cables, the LCU is installed in field of one or two group of three-phase HV apparatus. The current and voltage signals picked up through transducers are led into LCU and then coupled, conditioned, analog/ digital converted and processed. Measuring parameters are treated within the LCU. The distributed online monitoring system at present includes six kinds of LCU, for bushing, potential transformer (capacitor voltage transformer), metal oxide arrester, instrument transformer, transformer, ambient temperature and humidity. It is easy to integrate other online monitoring function such as DGA, partial Charge, moisture and pressure of SF6 gas chamber etc into the system.

The schematic diagram of the LCU is shown in Figure 4. First, the analog signals are passed through a signal conditioning circuit which provides the transient protection and isolation of the input analog signals. A 16-bit resolution ADC is used to implement simultaneous sampling of the current and voltage signals. Finally, the algorithm of the DFT is performed inside the DSP chip and the digital computation of the dielectric loss factor, capacitance and other parameters are ready for uploading to the substation control unit (SCU). In addition to the values of the dielectric loss factor and capacitance, the waveforms of the voltage, current, temperature, humidity and frequency signals and the harmonic components of the voltage and current can be displayed at SCU.

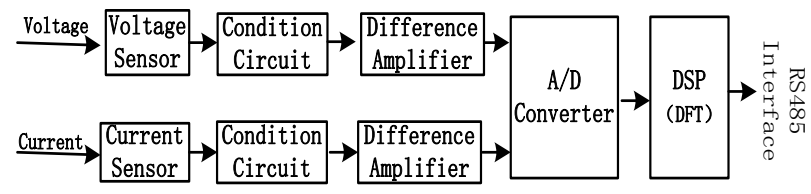

Figure 4. Schematic of the LCU

\subsection{Substation Control Unit}

A substation control unit (SCU), installed in the control cabinet, is the control center of the online monitoring system at the substation. The SCU can send out commands based on the communication protocol, such as start sampling, stop sampling, upload data, reset DSP etc., to control the operation of the LCU. And also, the SCU can process the LCU data to display all the monitoring results to engineers who may concern about the operation condition of the HV equipment.

A field RS485 bus is applied in the monitoring system for communication. It is in digital, multi spots and bi-directional communication mode. Relying on the flexible communication network a SCU can link with extendible LCU without exact number limit. The onsite twisted pair daisy chain RS485 network is connected through fiber-optic cable to the SCU. Compared with systems based on electrical cables, the approach of optical fiber communications has advantages, the most important of which are: fiber-optic cables are immune to problems of electrical cables such as ground loops or electromagnetic interference (EMI). High voltage signals are completely isolated between the onsite electrical cables and the control computer in the control room. 
In addition, the SCU monitors and manages the operation of the LCU. If some failures occurs with one or more LCU, the SCU will reset the LCU a couple of times to fix the problem even the malfunction of one or more LCUs will not affect the operation of the remaining LCUs in the online monitoring system.

\subsection{Integrate To the Online Monitoring System}

The objective of the online monitoring system is to meet development of the smart grid and adapt to the newly adopted power production management policy. The state grid corporation of china plans to build a fully covered system, two level of deployment and three level of application, through the construction of the system to standardize the system structure, sensor or device calibration, certification, installation, communication protocol etc., at the same time, so that to show all the information such as the data display, warning results, analysis, diagnosis, evaluation, forecast etc., at different level of application.

\subsubsection{Terms and Definitions}

Before to state the detail part or element of the overall online monitoring system, there are a few terms and definitions must to make it clear so that to help understand the system. CAC is short for the Condition Information Acquisition Controller, installed in the substation, which can connect all the monitoring device or sensors though industrial communication bus such as RS485 or Ethernet or serial port to from an online monitoring system in a substation. The $\mathrm{CAC}$ can receive all the information from the monitoring devices and send commands to control the process of the monitoring devices; CMU is short for the Comprehensive Monitoring Unit, installed in substation, which can receive the information from the monitoring devices or sensors, after process the raw data, it can communication with substation monitoring unit through standardized communication bus or interfaces; SMU is short for the Substation Monitoring Unit, installed in substation, which is responsible for the data analysis, administration of the CMU and monitoring devices, parameter setting, data summon, time synchronization, device reset etc, and also to communication with the CAG; CAG is short for the Condition Acquisition Gateway, arranged at the provincial utilities, which can communication with the CAC.

\subsubsection{System Structure}

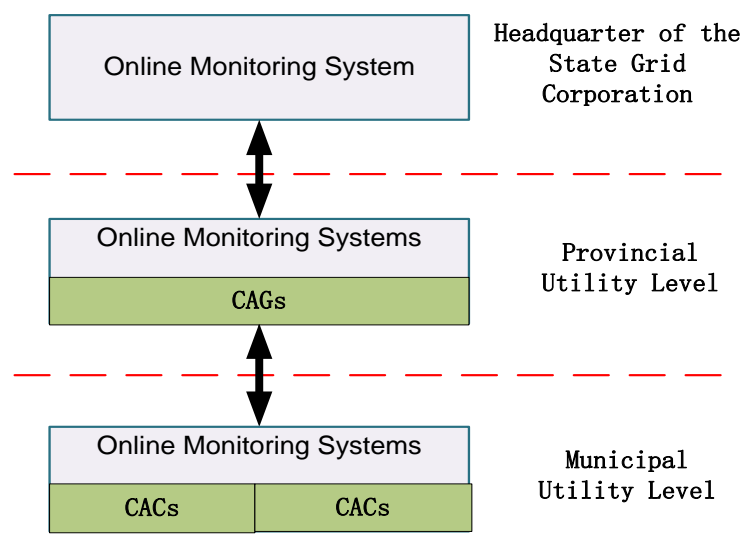

Figure 5. Framework of the Online Monitoring System 
The overall system structure as Figure 5 shows, two level of deployment and three level of application. The two level of deployment is to arrange the online monitoring system at the each province based utility and headquarter of the state grid corporation. The municipal utilities (substations) plan to arrange CACs (Condition Information Acquisition Controller) to connect all the online monitoring sensors or devices so that the municipal information can be transferred to the province and headquarter system. The three level of application is that the monitoring system can be used by municipal utilities and provincial utilities and headquarter of the state grid corporation.

\subsubsection{System Interface Classification}

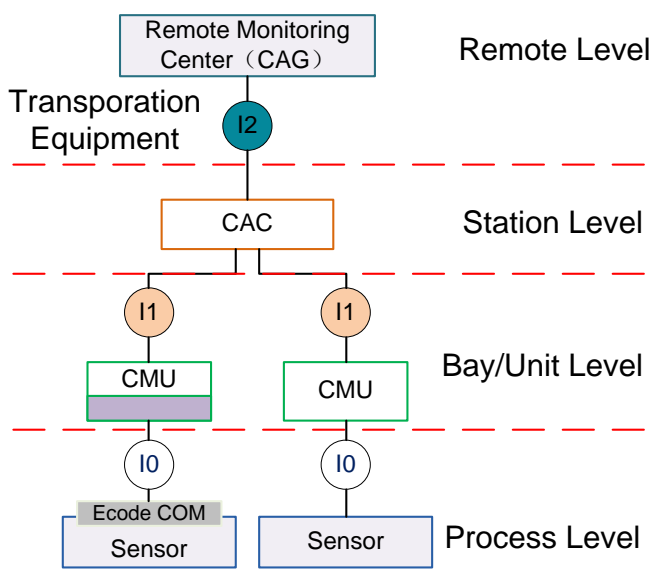

Figure 6. System Interface Classification

The substation online monitoring system as Figure 6 show has four levels, which are process level, bay/unit level, station level, and CAG level. To make the online monitoring system work smoothly, the information obtained from the monitoring transducers or devices transfers three communication protocols to the CAG. The three communication protocols are interfaces I0, I1, I2. Interface I0 is between the monitoring devices (or transducers) and $\mathrm{CMU}$, one way is to transfer the conditioned, A/D conversion, preconditioned signal to the $\mathrm{CMU}$, the other is send setting parameters, time synchronization command, data summon command, device reset etc., to the monitoring devices; Interface I1 is between the CMU and SMU. The CMU calculates the monitoring data through the calculation model, after that the CMU send the normalized data, analysis results, warning results to the SMU. The SMU can update the calculation models, summon data, time synchronization, reset etc., to the CMU; Interface I 2 is between the SMU and CAG. The SMU send the monitoring data, analysis results, warning information etc to the provincial based CAG. The SMU can also receive the analysis models, parameter configurations, data summon, time synchronization, device reset etc., from the CAG;

\subsubsection{Substation System Structure:}

The substation online monitoring system is a distributed and hierarchy structure as Figure 7 shows, which is composed of process level, unit/bay level, station level, remote monitoring center. 
The process level consists of all the monitoring devices or sensors of the transformers, reactors, circuit breakers, GISs (Gas Insulated Switchgear), MOAs (Metal Oxide Arrester), bushings etc. The monitoring devices including DGA (Dissolved Gas Analysis), PD (Partial Discharge), transformer oil and winding temperature, transformer core ground current, dielectric loss factor, temperature, moisture, pressure and density of the GIS cabinet etc.

The IEC61850 standard is the communication standard for the smart substation, which has been successfully used in the substation automation system. At the pilot stage of the smart substation project, not all of the online monitoring devices can comply with the IEC61850 standard, the CMU is adopted so that the online monitoring system can comply with the IEC61850, as the development of new monitoring devices which embedded with IEC61850 module, the CMU and unit/bay level can be omitted in future application.

The station level is the SMU, which controls the operation of the whole online monitoring system, and also collect all the monitoring results to analysis, diagnosis, warning, data display, data storage and forward.

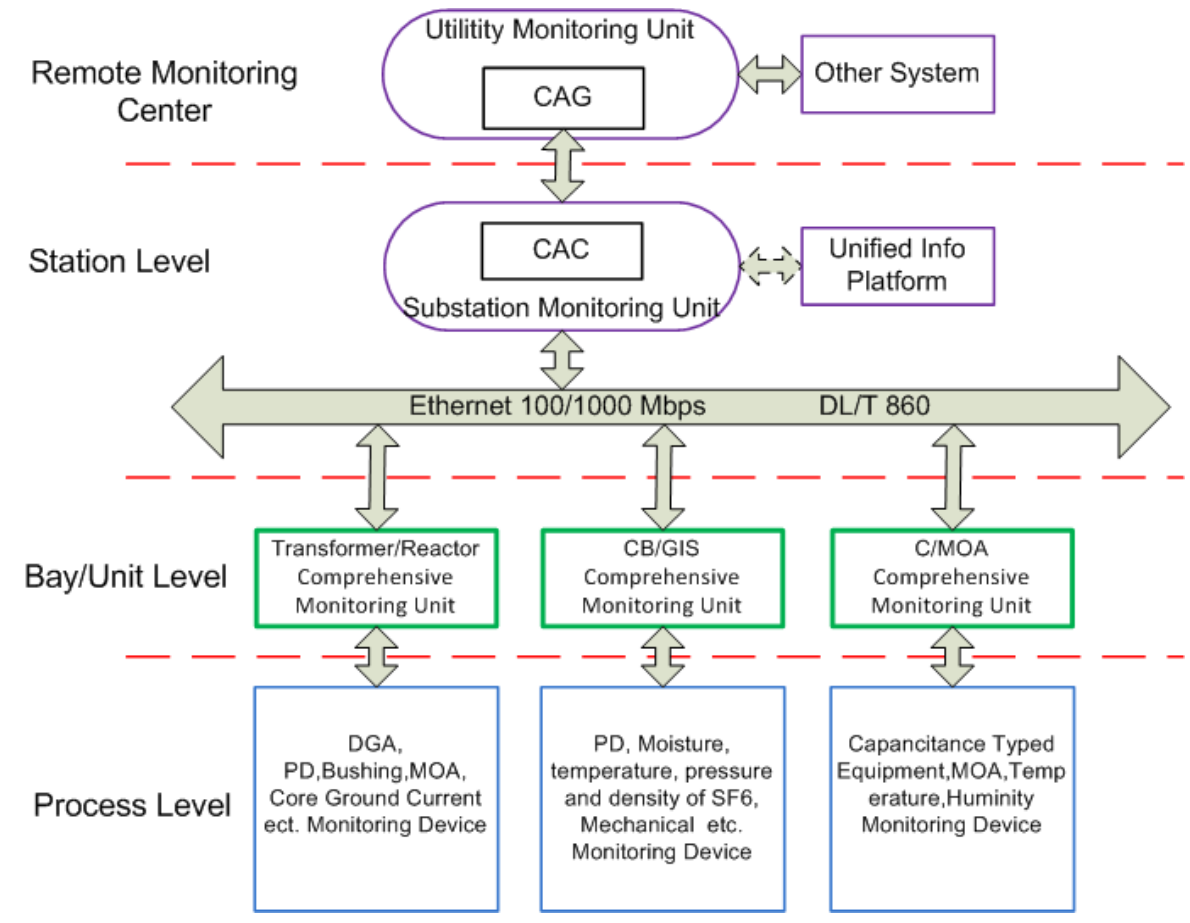

\section{Figure 7. The Schematic Structure of the Substation Online Monitoring System}

The remote monitoring center is also called provincial utility monitoring unit. In this paper, it is only focus on the transportation equipment and the system structure. The transportation online monitoring system as a sub system of the CAG, it can use the analysis models and methods to evaluate the condition of the high voltage equipment based on the online monitoring data or live measuring data. The data or analysis results based on the CAG platform can be published through web in the exclusive networks, and also it can offer the information to other system as foundation data of the asset condition evaluation and maintenance. 


\section{Ways to Improve Accuracy}

During the performance of the DFT on the detected data, non-simultaneous sampling and poor synchronization between sampling rate and signal period, indirect voltage reference, leakage current transducer design are believed to be the main source of error in the measurement of the magnitude and the phase of the signals. To improve the effectiveness of the DFT algorithm and measuring accuracy, four measures were taken in the design of the LCU.

\subsection{Simultaneous Sampling}

Although DSP or microprocessor inner A/D converter has multiple analog input channels, there is only one converter in the ADC module. It is very difficult to acquire the current and voltage at the same time. To ensure the synchronization of all the input analog signals, a special ADC which eliminates the necessity calibration of the time difference resulted from channel switching was adopted.

\subsection{Synchronization and Frequency Fluctuation Tracing}

In dielectric loss factor measuring procedure the information of fundamental phase angle of the current and voltage signals are extracted and calculated, however, the most important thing is to guarantee the $2^{N}$ sample dots with exactly the same interval dispersed in one network frequency period. The network frequency fluctuates around $50 \mathrm{~Hz}$, frequency trace is needed while sample $2^{N}$ fixed dots in order to confirm the actual sampling frequency. Because the fluctuating of network frequency is slowness, the LCU adopts pretty simple measuring frequency circuit to realize sample synchronization. By means of a voltage comparator, convert the sinusoidal reference voltage signal to square signal, then apply the square wave to the capture pin of the Event Manager (EM is one module of the DSP). Before start the conversion process of the ADC, DSP measure the time interval between the two adjacent rising edges or falling edges of the square wave signal, and the interval is the network period. After get the period of the network, the hardware program can dynamically set the sampling frequency of the ADC by the control of the DSP timer so that the $2^{N}$ sample dots are exactly acquired in one cycle. In this work, 128 sample dots were acquired in one period.

\subsection{Direct Voltage Reference}

Indirect voltage reference: To measure the dielectric loss factor, usually take the secondary voltage of a potential transformer as the reference voltage. The coil of the potential transformer is usually connected with power system protection and metering devices, no spare coil for the online monitoring system. For the safety concerns, usually a resistor is connected at the secondary terminal of potential transformer, the network voltage is converted into a current and can also gained by a current sensor with the same theory of the leakage current sensor. To reduce the effect of directly using the reference voltage another common way usually is that apply only one or two reference voltage to the LCU and supply a $220 \mathrm{~V}$ AC power to each LCU. Dielectric loss factor is derived indirectly by subtraction phase angle of the reference voltage versus power supply, leakage current versus power supply, 
$220 \mathrm{~V}$ AC power supply serve as a virtual reference to synchronize the voltage and current signals. Indirect voltage reference will definitely cause some phase shift to the dielectric loss factor measurement.

2) Direct voltage reference: To satisfy the safety and measuring requirements, metering coil of the potential transformer is used as reference voltage. And a resistor voltage divider with transient protection is adopted, the reference voltage is applied directly to each LCU with fuse and air circuit breaker protection. This method will avoid the phase shift caused by the reference voltage.

\subsection{Current Transducer Design}

As the dielectric loss factor is quite small, usually less than one percent, several conditions must be satisfied to obtain high measurement accuracy. The phase error of the current transducer is the main cause of measurement error. Traditionally, current transformer is the common way to pick up the leakage current. The excitation current is believed to cause some kinds of phase shift even with some improved methods, such as zero flux current transducer. In the past, some researchers connect a resistor or a capacitor at the terminal tap of the HV apparatus to sample the current signal. However, this is not accepted from the viewpoint of the utilities. To satisfy the safety in the utilization case, a coiled power resister with reliable safety protection transducer was designed as Figure 8 shows. This kind of transducer was installed more than $80 \mathrm{HV}$ equipment with more than three years of field operation, such as bushings, instrument transformers. As for transducer for MOA, to measure the leakage and resistive current, the dielectric loss angle is around 3 or 5 degree, the usual CT typed transducer can satisfy the measurement requirements. Figure 9 shows the diagram of the transducer for MOA.

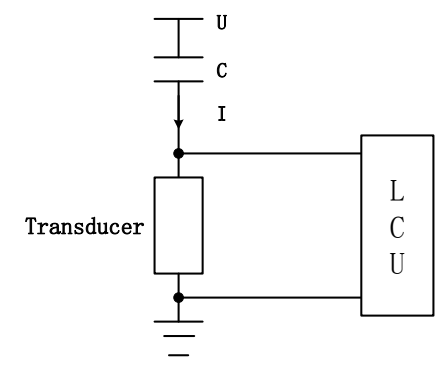

Figure 8. Resistor Transducer

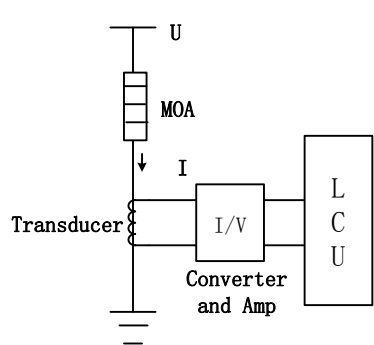

Figure 9. Transducer for MOA

\section{Field Operation Results}

The online monitoring systems were installed on three substations to evaluate the insulation of $\mathrm{HV}$ apparatus. There are totally around 18 bushings, 66 current transformers, 18 MOAs, three transformer earth currents, one transform DGA, etc. Figure 10 shows an illustration of the graphic user interface, and the readouts and display that it provides. 


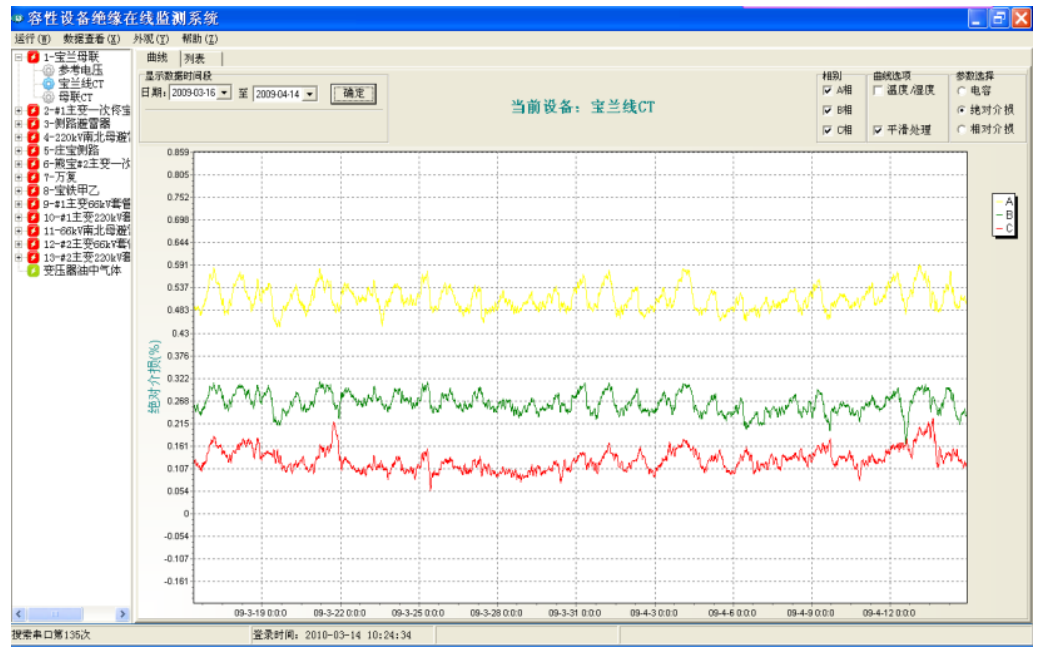

Figure 10. Graphic User Interface of the System

Figure 11 shows the continuous monitoring results of one group of the current transformer bushings in a whole month. The graph shows that $\tan \delta$ may fluctuate a little bit with the variation of temperature and humidity. The variation of the of the dielectric loss factor tan $\delta$ is ranging from 0.39 to 0.52 for phase $\mathrm{A}$, from 0.22 to 0.32 for phase $\mathrm{B}$, from 0.022 to 0.13 percent for phase $\mathrm{C}$.

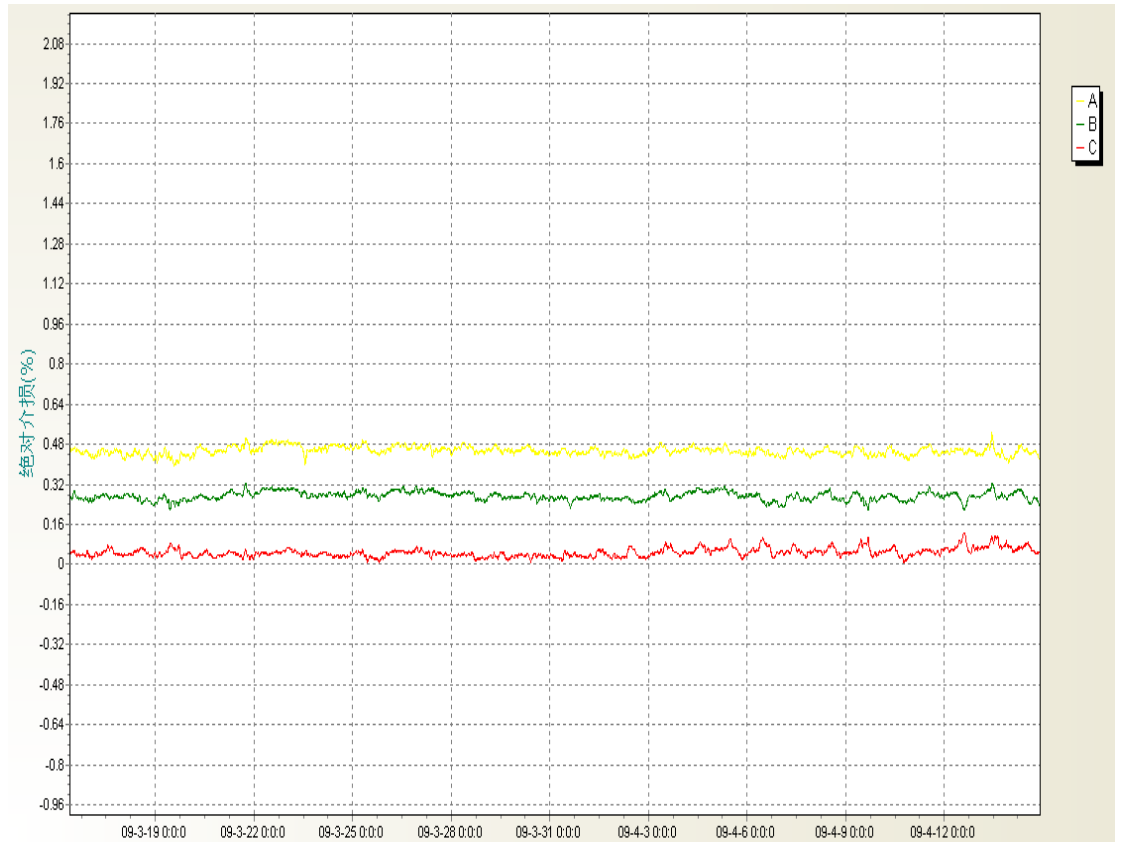

Figure 11. Continuous Monitoring Results of Current Transformer

\section{Summary}

The developed measuring unit of the on-line monitoring system can effectively improve the simultaneous sample between multi-channel voltage and current signals. And the dynamic sampling rate setting method can trace the frequency fluctuation and improve the synchronization between the sampling rate and signal period. The 
methods described in this paper not only can improve the effectiveness of the DFT algorithm, but also can withstand frequency fluctuation.

Furthermore, the direct reference voltage and sampling transducer designed by the author can further reduce the phase shift of the voltage and current signals. The protection and safety measures are proved reliable and safe in field operation. The distributed on-line monitoring system has high precision of measurement, strong ability of anti-interferences, easy to integrate other functions, flexible communication networks, steady and reliable running performance. The suitability of the developed system for on-line application has been demonstrated.

\section{References}

[1] B. Z. Shi, L Yang, X. Y. Jiang, H. B. Wang, and L. C. Zhou. Instrumentation of Online Insulation Monitoring of HV Apparatus. Proceedings of The 6th International Conference on Properties and Application of Dielectric Materials, 716 (2000)

[2] B. Z. Shi, L. Yang, X. Y. Jiang, H. B. Wang, and L. C. Zhou. An Online Insulation Diagnosis Technique for HV Apparatus Base on Pertinency. Proceedings of The 6th International Conference on Properties and Applications of Dielectric Materials, 708 (2000)

[3] Q. Gao, P. N. Ding, Y. Han and B. H. Geng. Development of Distributed On-line Monitoring System for Dielectric Loss Tangent of High Voltage Capacitive Apparatus. 2008 International Conference on Condition Monitoring and Diagnosis, Beijing, China, April 21-24, 1179 (2008)

[4] W. Pei, M. R. Raghuveer, W. McDermid, and J. C. Bromley. A Digital Technique for the On-line Measurement of Dissipation Factor and Capacitance. IEEE Transactions on Dielectrics and Electrical Insulation, 8, 228 (2001)

[5] H. P. Zhang, K. X. Tan, F. Y. Dong, and J. C. Wang. The Analysis of On-line Monitored Results for Capacitive Type of Equipment. ISEIM, 805 (2001)

[6] Yang Minzhong, Liu Shaoyu, Wang Zhuo and Yan Zhang, "Error Analysis for Dielectric Loss Measurement Based on Harmonic Anlysis", ISEIM, 336 (2001)

[7] Q/GDW Z410 - 2010 Technical Guide for Smart Electric Equipments

[8] Q / GDW 534-2010 Technical Guide for On-line Monitoring System of Transformation Equipment

[9] Q / GDW 393-2009 Specifications of design for 110(66) kV 220kV Smart Substation

[10] Q / GDW 616-2011 Application Specification for On-line Monitoring Device of Transformation Equipment Based on DL/T 860

[11] DL/T 860-2006 Communication Networks and Systems in Substations

\section{Authors}

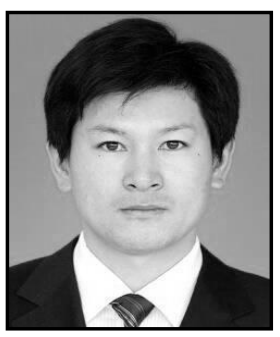

Gao Qiang, is a senior Engineer of the Liaoning Electric research Institute, Dean of R \& D department, Shenyang, China. $\mathrm{He}$ is studying for his $\mathrm{Ph}$. D degree at Shanghai Jiao tong University. His current research interests include online monitoring technology of high voltage equipment such as transformers, Metal oxide arrester, Circuit Breaker, GIS, bushing, underground cable etc. 


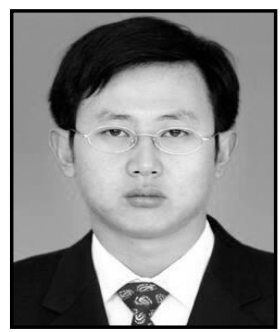

Geng Baohong, is a senior Engineer of the Liaoning Electric research Institute, Deputy director of $\mathrm{R} \& \mathrm{D}$ department, Shenyang, China. He is M.S. of Xi' an Jiao Tong University. His current research interests include online monitoring technology of high voltage equipment and application of IEC61850 standard.

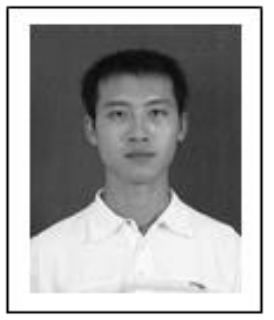

Zhang guangming is a $R \& D$ engineer of the Liaoning Electric research Institute, Shenyang, China. He received M.S. degree of pattern recognition and intelligent system from Northeastern University, China, in 2010. Currently he is major in diagnosis method for power equipment and design of intelligent electronic device. 